International Journal of Wavelets, Multiresolution

and Information Processing

Vol. 14, No. 6 (2016) 1699001 (5 pages)

1 World Scientific

(C) World Scientific Publishing Company

DOI: $10.1142 / S 0219691316990010$

www.worldscientific.com

\title{
Author index (Vol. 14)
}

Abbasi, R., see Zuo

Akçay, H. and Türkay, S., Spectrum estimation with missing values: A regularized nuclear norm minimization approach

Akila, L. and Roopkumar, R., Ridgelet transform for quarternion-valued functions

Andrianov, P. and Skopina, M., On Jackson-type inequalities associated with separable Haar wavelets

Ashino, R., see Bahri

Askari Hemmat, A., see Tahami

Atreas, N. D., Characterizations of dual multiwavelet frames of periodic functions

Bahri, M. and Ashino, R., Some properties of windowed linear canonical transform and its logarithmic uncertainty principle

Benediktsson, J. A., see Ye

Bhattacharjee, A. K., see $\mathrm{Si}$

Bin, Y., Chao, Y. and Guoyu, H., Efficient image fusion with approximate sparse representation

Birajdar, G. K. and Mankar, V. H., Passive method for rescale detection using quadrature mirror filter based higher order statistical features

Chao, Y., see Bin
14 (2016) 1650040

14 (2016) 1650054

14 (2016) 1650006

14 (2016) 1650005

14 (2016) 1650015

14 (2016) 1650004

14 (2016) 1650012

14 (2016) 1650015

14 (2016) 1640005

14 (2016) 1650041

14 (2016) 1650024

14 (2016) 1650033 14 (2016) 1650024
Chen, C. and Chen, N., Penalized empirical relaxed greedy algorithm for fixed design Gaussian regression

Chen, H., Learning rate of magnitudepreserving regularization ranking with dependent samples

Chen, N., see Chen

Chen, W., see Ye

Cheng, C. and Zhang, L., An efficient segmentation method based on dynamic graph merging

Cheng, Q., see Xiong

Cui, H., Liu, D., Li, Y. and He, H., A dynamic filtering obstacle detection method based on ultrasonic array

Cui, M., see Liu

Dastourian, B. and Janfada, M., Frames for operators in Banach spaces via semi-inner products

De, A., see $\mathrm{Si}$

Dehua, Z., see Shengan

Deng, H., see Wei

Dongfa, G., see Shengan

Fei, M., Mi, W. and Pan, Y., On model reduction with consecutively selected rational basis

Fei, M., Pan, Y. and $\mathrm{Xu}, \mathrm{Y}$., Some shaper uncertainty principles for multivector-valued functions

Fujimoto, S., see Zheng Fukuda, N., Kinoshita, T. and Suzuki, T.,
14 (2016) 1650019

14 (2016) 1650001

14 (2016) 1650019

14 (2016) 1650049

14 (2016) 1650052

14 (2016) 1650038

14 (2016) 1650030

14 (2016) 1650023

14 (2016) 1650011

14 (2016) 1650041

14 (2016) 1650025

14 (2016) 1640007

14 (2016) 1650025

14 (2016) 1650002

14 (2016) 1650043

14 (2016) 1650051 
On the unconditional convergence of wavelet expansions for continuous functions

Grohs, P., Kereta, Ž. and Wiesmann, U., A shearlet-based fast thresholded Landweber algorithm for deconvolution

Gülerce, M. and Ünal, G., Using wavelet analysis to uncover the co-movement behavior of multiple energy commodity prices

Guo, Q. and Ye, P., Coefficient-based regularized regression with dependent and unbounded sampling

Guoyu, H., see Bin

Hashim, M. H. A., see Mahmoud

He, H., see Cui

He, M., see Nian

$\mathrm{Hu}$, C.-M., see Zhou

Huang, K.-K., see Liu

Huang, W., see Liu

Huang, Z.-H., see Li

Janfada, M., see Dastourian

Jansen, M., Nonequispaced B-spline wavelets

Jha, N. K., see Pandey

Josyula, K. L. S. J., see Kumar

Kanchana, M. and Varalakshmi, P., Computer aided system for breast cancer in digitized mammogram using shearlet band features with LS-SVM classifier

Kang, M., see Sáfadi

Kereta, Ž., see Grohs

Kinoshita, T., see Fukuda

Kong, Y., see Xiong

Kou, K. I. and $\mathrm{Li}$, H., Greedy adaptive decomposition of signals based on nonlinear Fourier atoms

Kou, K. I., see Song

Krivoshein, A. V., Multivariate symmetric
14 (2016) 1650007

14 (2016) 1650032

14 (2016) 1650047

14 (2016) 1650039

14 (2016) 1650024

14 (2016) 1650044

14 (2016) 1650030

14 (2016) 1650029

14 (2016) 1650022

14 (2016) 1650021

14 (2016) 1650023

14 (2016) 1650035

14 (2016) 1650011

14 (2016) 1650056

14 (2016) 1650037

14 (2016) 1650008

14 (2016) 1650017 14 (2016) 1650027 14 (2016) 1650032

14 (2016) 1650007 14 (2016) 1650038

14 (2016) 1650014 14 (2016) 1640004 refinable functions and function vectors

Kumar, M. and Josyula, K. L. S. J., An interlaced secure algorithm for RGB image encryption in wavelet packet domain

Kumar, P., see Prasad

Leite, I. C. C., see Sáfadi

Li, H., see Kou

Li, H., see Liu

Li, H., see Song

Li, H., see Ye

Li, L., Yang, C. and Xie, Q., 1D embedding multi-category classification methods

Li, L., see Wang

$\mathrm{Li}, \mathrm{X}$., see Zuo

Li, Y., see Cui

Li, Z.-M., Huang, Z.-H. and Zhang, T., Gaborscale binary pattern for face recognition

Lian, Q., see You

Liao, F., see Wu

Liu, D., Wu, J. and $\mathrm{Xu}, \mathrm{X}$. , Multi-agent reinforcement learning using ordinal action selection and approximate policy iteration

Liu, D., see Cui

Liu, H. and Huang, K.K., Zerotree wavelet image compression with weighted subblock-trees and adaptive coding order

Liu, L., Zhou, Y., Li, H., Huang, W. and Cui, M., A novel infrared moving target detection algorithm based on multiscale codebook model

Liu, Y., Wu, W. and Xu, G., The best constants in the Wirtinger inequality

Long, H. and Yan, G., Forecasting import and export volume with a combined model based on wavelet filtering

Mabrouk, A. B., see Mahmoud
14 (2016) 1650034

$\begin{array}{ll}\mathbf{1 4}(2016) & 1650008 \\ \mathbf{1 4}(2016) & 1650046 \\ \mathbf{1 4}(2016) & 1650027 \\ \mathbf{1 4}(2016) & 1650014 \\ \mathbf{1 4}(2016) & 1650023 \\ \mathbf{1 4}(2016) & 1640004 \\ \mathbf{1 4}(2016) & 1640005\end{array}$

14 (2016) 1640006

14 (2016) 1640003

14 (2016) 1650040

14 (2016) 1650030

14 (2016) 1650035

14 (2016) 1650055

14 (2016) 1650045

14 (2016) 1650053

14 (2016) 1650030

14 (2016) 1650021

14 (2016) 1650023

14 (2016) 1650048

14 (2016) 1650018

14 (2016) 1650044 
Mahmoud, M. M. I., Mabrouk, A. B. and Hashim, M. H. A., Wavelet multifractal modeling and prediction of transmembrane proteins series

Mankar, V. H., see Birajdar

Mi, W., see Fei

Mirzaee Azandaryani, M., Erasures and perturbations of g-frames and fusion frames in Hilbert $C^{*}$-modules

$\mathrm{Mo}, \mathrm{X}$. and $\mathrm{Xu}, \mathrm{J}$, , Convergence and consistency of ERM algorithm with uniformly ergodic Markov chain samples

Nian, Y., Xu, K., Wan, J., Wang, L. and He, M., Block-based KLT compression for multispectral images

Pan, Y., see Fei

Pan, Y., see Fei

Pandey, J. N., Jha, N. $K$. and Singh, $O$. P., The continuous wavelet transform in $n$-dimensions

Poria, A., Behavior of Gabor frame operators on Wiener amalgam spaces

Prasad, A. and Kumar, P., The continuous fractional wavelet transform on a generalized Sobolev space

Rinoshika, A., see Zheng

Roopkumar, R., see Akila

Sáfadi, T., Kang, M., Leite, I. C. C. and Vidaković, B. Wavelet-based spectral descriptors for detection of damage in sunflower seeds

Saha Ray, S., see Sahu

Sahu, P. K. and Saha Ray, S., A numerical approach for solving nonlinear fractional Volterra-Fredholm integro-differential equations with mixed boundary conditions

Shen, Z. W., Construction of symmetric fractional overcomplete wavelets and applications in image restoration

14 (2016) 1650033

14 (2016) 1650002

14 (2016) 1650003

14 (2016) 1650013

14 (2016) 1650029

14 (2016) 1650002

14 (2016) 1650043

14 (2016) 1650037

14 (2016) 1650028

14 (2016) 1650046

14 (2016) 1650051

14 (2016) 1650006

14 (2016) 1650027 14 (2016) 1650036
Shengan, Z., Dongfa, G. and Dehua, Z., Quick draw of the original handwriting base on quadratic Bezier curve

Si, T., De, A. and Bhattacharjee, A. K., MRI brain lesion segmentation using generalized opposition-based glowworm swarm optimization

Singh, O. P., see Pandey

Skopina, M., see Andrianov

Song, Y., Li, H., Wang, J. and Kou, K. I., Multiple one-dimensional embedding clustering scheme for hyperspectral image classification

Song, Y., see Ye

Srivastava, S. and Yadav, G. C. S., On fixed point sets of wavelet induced isomorphisms and frame induced monomorphisms

Srivastava, S. and Yadav, G. C. S., On wavelet induced isomorphisms for reducing subspaces

Suzuki, T., see Fukuda

Tahami, M., Askari Hemmat, A. and Yousefi, S. A., Numerical solution of twodimensional first kind Fredholm integral equations by using linear Legendre wavelet

Tang, P., see Zhou

Tang, Y. Y., see Wang

Tang, Y. Y., see Wu

Tang, Y., see Zuo

Toda, H. and Zhang, Z., Orthonormal basis
14 (2016) 1650036

14 (2016) 1650020

14 (2016) 1650025

14 (2016) 1650041

14 (2016) 1650037

14 (2016) 1650005

14 (2016) 1640004

14 (2016) 1640005

14 (2016) 1650016

14 (2016) 1650009

14 (2016) 1650007

14 (2016) 1650004

14 (2016) 1650022

14 (2016) 1640003

14 (2016) 1650045

14 (2016) 1650040 
of wavelets with customizable frequency bands

Toda, H. and Zhang, Z., Orthonormal wavelet basis with arbitrary real dilation factor

Türkay, S., see Akçay

Ünal, G., see Gülerce

Varalakshmi, P., see Kanchana

Vidaković, B., see Sáfadi

Wan, J., see Nian

Wang, C., see Ye

Wang, J., see Song

Wang, J., see Wang

Wang, J., see Ye

Wang, J., see Zhang

Wang, J., Semisupervised learning using ensembles of multiple 1Dembedding-based label boosting

Wang, J., Semisupervised learning using multiple onedimensional embedding based adaptive interpolation

Wang, L., see Nian

Wang, W., see Zhang

Wang, Y., Tang, Y. Y., Li, L. and Wang, J., Face recognition via collaborative representation based multiple one-dimensional embedding

Wang, Y., Laws of the iterated logarithm for intersections of Wiener sausages in critical dimensions

Wei, Y., You, X. and Deng, H., Small infrared target detection based on image patch ordering

Wen, J., see Xiong

Wiesmann, U., see Grohs

Wu, J., Liao, F. and Tang, Y. Y., Applications of the preview control method to the optimal problem for linear continuoustime stochastic systems with time-delay
14 (2016) 1650050

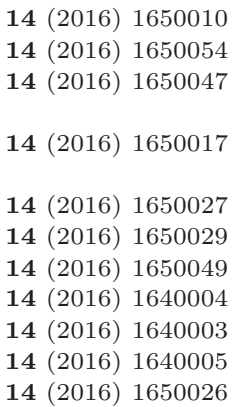

14 (2016) 1640001

14 (2016) 1640002

14 (2016) 1650029

14 (2016) 1650026

14 (2016) 1640003

14 (2016) 1650042

14 (2016) 1640007

14 (2016) 1650038

14 (2016) 1650032

14 (2016) 1650045
$\mathrm{Wu}$, J., see Liu

$\mathrm{Wu}, \mathrm{W}$., see Liu

Xiang, Z.-Q., A note on the stability of gframes in Hilbert $C^{*}$ modules

Xiao, J., see Zuo

Xie, Q., see Li

Xiong, X., Cheng, Q., Kong, Y. and Wen, J., A wavelet method for numerical fractional derivative with noisy data

$\mathrm{Xu}, \mathrm{G}$., see Liu

$\mathrm{Xu}$, J., see Mo

$\mathrm{Xu}, \mathrm{K}$., see Nian

$\mathrm{Xu}$, L., see Zuo

$\mathrm{Xu}, \mathrm{X}$, see Liu

$\mathrm{Xu}, \mathrm{X}$., see $\mathrm{Ye}$

$\mathrm{Xu}, \mathrm{Y}$., see Fei

Yadav, G. C. S., see Srivastava

Yadav, G. C. S., see Srivastava

Yan, G., see Long

Yang, C., see Li

Yang, J., see You

Ye, L., Wang, C., Xu, X. and Chen, W., Multiclass $\ell_{2}$-Boost with the scoring coding

Ye, P., see Guo

Ye, Z., Li, H., Song, Y., Wang, J. and Benediktsson, J. A., A novel semi-supervised learning framework for hyperspectral image classification

You, M., Yang, J. and Lian, Q., Wilson frames for $\mathbb{C}^{L}$ with general lattices

You, X., see Wei

Yousefi, S. A., see

Tahami nums14120161650004

Zhang, J., Wang, J. and Wang, W., A perturbation analysis of block-sparse compressed sensing via mixed $\ell_{2} / \ell_{1}$ minimization

Zhang, L., see Cheng

Zhang, T., see $\mathrm{Li}$

Zhang, Z., see Toda

Zhang, Z., see Toda

Zhang, Z., see Zhou

Zheng, Y., Rinoshika, A. and Fujimoto,
14 (2016) 1650053

14 (2016) 1650048

14 (2016) 1650031

14 (2016) 1650040

14 (2016) 1640006

14 (2016) 1650038

14 (2016) 1650048

14 (2016) 1650013

14 (2016) 1650029

14 (2016) 1650040

14 (2016) 1650053

14 (2016) 1650049

14 (2016) 1650043

14 (2016) 1650009

14 (2016) 1650016

14 (2016) 1650018

14 (2016) 1640006

14 (2016) 1650055

14 (2016) 1650049

14 (2016) 1650039

14 (2016) 1640005

14 (2016) 1650055

14 (2016) 1640007

14 (2016) 1650026

14 (2016) 1650052

14 (2016) 1650035

14 (2016) 1650010

14 (2016) 1650050

14 (2016) 1650022 
S., Two-dimensional orthogonal wavelet multi-resolution analysis on multi-scale flow structures behind triangle cylinder

Zhou, Y., see Liu

Zhou, Z.-G., Hu, C.-M., Tang, P. and Zhang, Z., Monitoring abrupt changes in satellite time series by seasonal confidence interval of regression residuals

14 (2016) 1650022
14 (2016) 1650051 14 (2016) 1650023
Zuo, X., Li, X., Xiao, J., Tang, Y., Abbasi, R. and $\mathrm{Xu}, \mathrm{L}$., Linear complexity of generalized cyclotomic binary sequences of order $2 d$ and length $2 p^{m}$

14 (2016) 1650040 